\title{
Study of Conjunctival Microbial Flora in Patients of Intensive Care Unit
}

\author{
Kadambari Ramani ${ }^{1}$, Subashini Kaliaperumal', Sandip Sarkar', Sujatha Sistla ${ }^{2}$ \\ ${ }^{I}$ Department of Ophthalmology, Jawaharlal Institute of Post Graduate Medical Education and Research, Puducherry, India \\ ${ }^{2}$ Department of Microbiology, Jawaharlal Institute of Post Graduate Medical Education and Research, Puducherry, India
}

\begin{abstract}
Purpose: The objective of the study was to evaluate the type of conjunctival microbial flora in intensive care unit patients and their antimicrobial sensitivity pattern.

Methods: A total of 272 samples (conjunctival swabs) were taken from patients in various intensive care units and sent for culture and sensitivity. An ocular examination was done to look for lagophthalmos, conjunctival discharge, exposure keratitis, and corneal perforation.

Results: Majority (82.1\%) of the samples showed at least one microbial isolate while 29 (10.7\%) samples showed multiple microbial growth. The most common microbes were coagulase negative Staphylococcus spp. (41.5\% of isolates), diphtheroids (11.0\% of isolates), and Staphylococcus aureus (9.6\% of isolates) which are the usual commensals of the ocular surface. Of the other microbes isolated, Pseudomonas aeruginosa (4.0\%) was the most common. Eighty-four percent isolates of coagulase negative Staphylococcus sp., $81.8 \%$ isolates of diphtheroids and 100\% isolates of Staphylococcus aureus were penicillin resistant. All isolates of Enterococcus fecalis were sensitive only to vancomycin. Two hundred and twenty eyes (80.9\%) had varying degrees of lagophthalmos. Nineteen (7.0\%) had severe corneal exposure changes leading to infectious corneal ulcer and perforation in all of them.

Conclusions: The isolates in patients of intensive care units were no different from the normal conjunctival flora though few pathogenic organisms such as Pseudomonas aeruginosa and Acinetobacter sp. were also isolated. Most of the isolates were penicillin resistant. This knowledge will help take appropriate prophylactic measures to contain ocular infections in the intensive care units.
\end{abstract}

Key Words: Conjunctiva, Drug resistance, Eye manifestations, Intensive care units, Microbiology

Received: December 8, 2020 Final revision: May 31, 2021

Accepted: June 24, 2021

Corresponding Author: Subashini Kaliaperumal, MS, DNB, FRCS (Glasg), MNAMS, FIMSA. Department of Ophthalmology, Jawaharlal Institute of Post Graduate Medical Education and Research, Gorimedu, Dhanvantari Nagar, Puducherry 605006, India. Tel: 91-759-850-0133, Fax: 91-413-229-6000, E-mail: subashini.k@jipmer.edu.in
The composition of the normal human ocular surface flora includes Staphylococcus epidermidis, Staphylococcus aureus, and diphtheroids. There are several factors that may alter the composition of ocular surface flora: age, dry eye, immunosuppressive medication and medical illnesses such as diabetes mellitus [1,2]. Patients in the intensive care unit (ICU) frequently have impaired ocular defence mech- 
anisms as a result of metabolic derangements, mechanical ventilation, sedation, paralysis and decreased level of consciousness [2]. The impaired ocular defence mechanisms include poor eyelid closure, inhibition of Bell's phenomenon, decreased blink reflex, reduced tear production $[3,4]$. The ICU also presents an environment rich in pathogens that may contribute in the increased exposure of the ocular surface to micro-organisms with significant antimicrobial resistance resulting from the widespread use of multiple antibiotics [2,5]. Moreover, prolonged eye closure which occurs in ICU patients causes a cascade of biochemical, cellular and microbial events culminating in inflammation, hypoxia and dry eye states [6]. Dry eye is one of the major risk factors of infectious keratitis [7]. The rate of positive conjunctival swab cultures in dry eye patients increases when compared with normal control, and the mean bacterial count is positively correlated with the reduction in goblet cells [8]. Eyelid closure during sleep is normally an active process involving contraction and relaxation of eyelid muscles. This active contraction and relaxation are lost with heavy sedation and use of muscle relaxants in ICU patients $[9,10]$. The blink reflex is also lost. Incomplete lid closure leads to drying of the mucosal surface and desiccation of the corneal epithelial tissues. All these factors increase the risk of microbial infections. The cornea is affected most often, with the most severe lesion being bacterial keratitis that may result in corneal opacity and blindness [9,11]. There are no large-scale studies on conjunctival microbial flora in particular on ICU patients. Hence this study was planned to determine the type of conjunctival microbial flora and their antibiotic sensitivity pattern in patients of ICUs.

\section{Materials and Methods}

The study of conjunctival microbial flora in ICU patients was carried out after approval from the Institution Ethics Committee and Scientific Advisory Committee of Jawaharlal Institute of Post Graduate Medical Education and Research (JIP/IEC/SC/2014/4/574) and adhered the tenets of the Declaration of Helsinki. The study was conducted between June 2014 and August 2014. A written informed consent was obtained from the patient or from the next of kin.

Adult patients aged more than 18 years with systemic ill- ness from medicine ICU, surgery ICU, casualty ICU, coronary ICU, and neurology ICU in a multidisciplinary tertiary referral centre of South India were included in the study. Patients with recent ocular trauma and concomitant ocular surface disease were excluded from the study.

The standard eye care for all sedated ICU patients included administering lubricating eye drops and eye patching was performed by the nursing staff. Initially, the patient's corneas were inspected grossly for exposure keratitis, corneal ulcer and corneal perforation using a penlight. Then superficial punctate keratopathy was ruled out by using fluorescein strip placed on the lower conjunctival sac and viewing through a hand held portable slit lamp using cobalt blue filter light. Patients were also examined for the presence or absence of lagophthalmos, con-

Table 1. Baseline demographics of the patients

\begin{tabular}{lc}
\hline Parameter & Value \\
\hline No. of patients & 136 \\
Age (yr) & \\
Mean \pm SD (range) & $42.68 \pm 17.21(23-79)$ \\
$<20$ & 15 \\
$20-40$ & 46 \\
$41-60$ & 57 \\
$>60$ & 18 \\
Sex & \\
Male & $78(57.35)$ \\
Female & $58(42.64)$ \\
Duration of stay (day) & \\
Mean \pm SD (range) & $4.5(1-13)$ \\
$<10$ & $109(80.14)$ \\
$\geq 10$ & $27(19.85)$ \\
Primary illness &
\end{tabular}

Values are presented as number or number (\%) unless otherwise indicated.

$\mathrm{SD}=$ standard deviation; $\mathrm{OP}=$ organophosphorus . 
junctival discharge, blepharitis and lid infections. Details such as age, sex, duration of stay and the underlying disease condition of each patient were collected. The procedure included collection of conjunctival swabs from both the eyes of the patient. Culture swab samples were taken from the lower conjunctival sac without touching the eyelid margin or lashes. Samples were taken from both the eyes and sent to culture room of microbiology laboratory as soon as possible. Using standard laboratory techniques, isolation, identification and antibiotic susceptibility of microbes was done.

The type of microbe and the antibiotic sensitivity were analysed using descriptive statistics and represented as frequencies and percentages using IBM SPSS Statistics ver. 20 (IBM Corp., Armonk, NY, USA). The presence of conjunctival discharge and corneal changes were also represented as frequencies and percentages. All statistical analyses were carried out at $5 \%$ level of significance and $p$-value $<0.05$ was considered as significant.

\section{Results}

Table 1 showing baseline characteristics of all the patients. we have recruited 136 patients from different ICUs, among them $76(55.88 \%)$ were male and $60(44.12 \%)$ were female. Mean age of the patients was $42.68 \pm 17.21$ years (range, 23-79 years) majority of them were in the 40 to 60 years age group with a male preponderance. Mean duration of stay of the patients was $6.9 \pm 4.5$ days (range, $1-13$ days).
A total of 272 samples were collected from 136 patients,. Among 272 collected conjunctival swab samples 76 (27.9\%) showed no microbial growth, in 196 (72.1\%) samples at least one microbe was isolated. Among the positive cultures, 225 microbial isolates were found and 29 (10.7\%) samples showed more than one microbial isolate. Majority of the isolates were the coagulase-negative Staphylococcus spp.

From medicine ICU, 148 samples were collected. Common cases included sepsis/urosepsis, OP poisoning, acute respiratory disease syndrome (ARDS), pneumonia, encephalitis and snake bite. Thirty-eight (25.7\%) samples showed no microbial growth. At least one microbe was isolated from 110 (74.3\%) samples. From the 110 samples that showed microbial growth, 129 microbial isolates were found. Nineteen (12.8\%) samples showed more than one microbial isolate.

From surgery ICU, 68 samples were collected. Common cases included bleeding gastric ulcer, perforation peritonitis, acute pancreatitis and acute cholecystitis. Twenty-four (35.3\%) samples showed no microbial growth. At least one microbe was isolated from $44(64.7 \%)$ samples. From the 44 samples that showed microbial growth, 52 microbial isolates were found. Eight (11.8\%) samples showed more than one microbial isolate. In most cases, ocular colonisation and systemic infection by the same bacteria co-existed in the same patient.

From casualty ICU, 28 samples were collected. Common cases included snake bite, organophosphorus poisoning and alcohol toxicity. Seven (25.0\%) samples showed no microbial growth. At least one microbe was isolated from 21

Table 2. Distribution of microbial isolates by microbe and ICU

\begin{tabular}{|c|c|c|c|c|c|c|}
\hline Microbe isolated & Total & Medicine ICU & Surgery ICU & Casualty ICU & Cardiology ICU & Neurology ICU \\
\hline CONS & $125(55.5)$ & $66(51.2)$ & $33(68.8)$ & $12(52.2)$ & $6(75)$ & $8(61.5)$ \\
\hline Diphtheroids & $33(14.67)$ & $16(12.4)$ & $3(6.3)$ & $7(30.4)$ & $2(25)$ & $5(38.5)$ \\
\hline $\begin{array}{l}\text { Staphylococcus } \\
\text { aureus }\end{array}$ & $29(12.89)$ & $19(14.7)$ & $10(20.8)$ & 0 & 0 & 0 \\
\hline $\begin{array}{l}\text { Pseudomonas } \\
\text { aeruginosa }\end{array}$ & $12(5.33)$ & $10(7.6)$ & 0 & $2(8.7)$ & 0 & 0 \\
\hline Acinetobacter sp. & $10(4.44)$ & $8(6.2)$ & 0 & $2(8.7)$ & 0 & 0 \\
\hline $\begin{array}{l}\text { Enterococcus } \\
\text { fecalis }\end{array}$ & $10(4.44)$ & $6(4.7)$ & $4(8.3)$ & 0 & 0 & 0 \\
\hline Candida sp. & $6(2.66)$ & $4(3.1)$ & $2(4.2)$ & 0 & 0 & 0 \\
\hline Total & $225(100)$ & $129(100)$ & $52(100)$ & $23(100)$ & $8(100)$ & $13(100)$ \\
\hline
\end{tabular}

Values are presented as number $(\%)$.

ICU = intensive care unit; CONS = coagulase negative Staphylococcus spp. 
Table 3. Microbial distribution based on primary illness

\begin{tabular}{|c|c|c|c|c|c|c|c|c|c|}
\hline Microbe & Sepsis & Poisoning & Peritonitis & Cardiac & Respiratory & Neurological & Infection & Miscellaneous & Total \\
\hline No growth & 7 & 8 & 2 & 5 & 12 & 6 & 10 & 26 & 76 \\
\hline CONS & 12 & 30 & 6 & 7 & 5 & 15 & 16 & 34 & 125 \\
\hline $\begin{array}{l}\text { Staphylococcus } \\
\text { aureus }\end{array}$ & 6 & 6 & 0 & 0 & 1 & 2 & 4 & 10 & 29 \\
\hline Candida spp. & 0 & 0 & 0 & 0 & 2 & 0 & 2 & 2 & 6 \\
\hline $\begin{array}{l}\text { Pseudomonas } \\
\text { aeruginosa }\end{array}$ & 0 & 0 & 0 & 0 & 3 & 0 & 8 & 1 & 12 \\
\hline $\begin{array}{l}\text { Acinetobacter } \\
\text { spp. }\end{array}$ & 0 & 0 & 0 & 0 & 3 & 2 & 4 & 1 & 10 \\
\hline Diphtheroids & 7 & 2 & 2 & 4 & 4 & 7 & 2 & 5 & 33 \\
\hline $\begin{array}{l}\text { Enterococcus } \\
\text { fecalis }\end{array}$ & 2 & 0 & 0 & 0 & 2 & 0 & 4 & 2 & 10 \\
\hline
\end{tabular}

Values are presented as number.

CONS = coagulase negative Staphylococcus spp.

(75.0\%) samples. From the 21 samples that showed microbial growth, 23 microbial isolates were found. Two (7.1\%) samples showed more than one microbial isolate.

From cardiac ICU, 12 samples were collected. The most common cases were myocardial infarction, congestive cardiac failure and unstable angina. Four (33.3\%) samples showed no microbial growth. All 8 (66.7\%) samples showed single isolates.

From neurology ICU, 16 samples were collected. The most common cases were ischemic stroke, subdural hematoma and intracerebral hemorrhage. Three (18.8\%) samples showed no microbial growth. From the 13 samples that showed microbial growth, 13 microbial isolates were found. Only normal commensals such as coagulase negative Staphylococcus spp. (CONS; $\mathrm{n}=8,61.5 \%$ ) and diphtheroids $(\mathrm{n}=5,38.5 \%)$ were isolated.

Table 2, 3, and 4 demonstrating the organism distribution based on ICU, primary illness and their antibiotics sensitivity pattern, respectively.

\section{Anti-microbial sensitivity}

\section{1) $\mathrm{CONS}$}

A total of 125 isolates of CONS were tested for antimicrobial sensitivity against ciprofloxacin, cloxacillin, erythromycin, gentamicin, penicillin and clindamycin. Of this 125, $17(13.6 \%)$ isolates were resistant to both erythromycin and gentamicin but sensitive to tetracycline while, 14
Table 4. Antimicrobial sensitivity of coagulase negative Staphylococcus spp.

\begin{tabular}{lccr}
\hline Antimicrobial agent & Sensitive & Intermediate & Resistant \\
\hline Ciprofloxacin & $96(76.8)$ & $6(4.8)$ & $23(18.4)$ \\
Cloxacillin & $65(52.0)$ & $2(1.6)$ & $58(46.4)$ \\
Erythromycin & $87(69.6)$ & $2(1.6)$ & $36(28.8)$ \\
Gentamicin & $104(83.2)$ & 0 & $21(16.8)$ \\
Penicillin & $20(16.0)$ & 0 & $105(84.0)$ \\
Clindamycin & $98(78.4)$ & $7(5.6)$ & $20(16.0)$ \\
\hline
\end{tabular}

Values are presented as number (\%).

$(11.2 \%)$ isolates were resistant to erythromycin, gentamicin, cloxacillin and clindamycin. They were sensitive to both tetracycline and vancomycin.

\section{2) Staphylococcus aureus}

A total of 29 isolates of Staphylococcus aureus were found. They were tested for antimicrobial sensitivity against ciprofloxacin, cloxacillin, erythromycin, gentamicin, penicillin and clindamycin. All the 29 (100\%) isolates were resistant to penicillin, but all of them were sensitive to ciprofloxacin, cloxacillin, erythromycin, gentamicin and clindamycin. 
Table 5. Microbial pattern associated with ocular findings

\begin{tabular}{lcccc}
\hline Microbe isolated & Conjunctival discharge & Exposure keratitis & Corneal perforation & Resistant \\
\hline CONS & 2 & 51 & 2 & 1 \\
Diphtheroids & 0 & 13 & 1 & 0 \\
Staphylococcus aureus & 9 & 29 & 4 & 8 \\
Pseudomonas aeruginosa & 10 & 12 & 5 & 0 \\
Acinetobacter sp. & 7 & 10 & 4 & 0 \\
Candida & 4 & 6 & 4 & 0 \\
Enterococcus faecalis & 4 & 10 & $19(7)$ & $2(0.7)$ \\
Total & $24(8.8)$ & $220(80.9)$ & & 0 \\
\hline
\end{tabular}

Values are presented as number or number (\%).

CONS = coagulase negative Staphylococcus spp.

\section{3) Pseudomonas aeruginosa}

Twelve isolates of Pseudomonas aeruginosa were found. They were tested for antimicrobial sensitivity against amikacin, ciprofloxacin, ceftazidime, cefoperazone plus sulbactam and gentamicin. All $12(100 \%)$ isolates were sensitive to all the mentioned antimicrobial agents.

\section{4) Acinetobacter spp.}

Ten isolates of Acinetobacter spp. were found. They were tested for antimicrobial sensitivity against amikacin, ciprofloxacin, ceftazidime, cefoperazone plus sulbactam and gentamicin. All $10(100 \%)$ isolates were sensitive to all the mentioned antimicrobial agents.

\section{5) Diphtheroids}

A total of 33 isolates of diphtheroids were found and tested for antimicrobial sensitivity against ciprofloxacin, cloxacillin, erythromycin, gentamicin, penicillin and clindamycin, of which 27 (81.8\%) of the isolates were resistant to penicillin. Only six (18.2\%) of the isolates were sensitive to penicillin. All the 33 isolates were sensitive to ciprofloxacin, cloxacillin, erythromycin, gentamicin and clindamycin.

\section{6) Enterococcus fecalis}

Ten isolates of Enterococcus fecalis were found. They were tested for antimicrobial sensitivity against ampicillin, tetracycline and vancomycin. All 10 isolates were resistant to both ampicillin and tetracycline. All the isolates were sensitive to vancomycin.

\section{Ocular findings in ICU patients}

Two hundred and twenty eyes (80.9\%) had varying degrees of lagophthalmos resulting in mild exposure keratitis. Twenty-four eyes (8.8\%) had conjunctival discharge, two $(0.7 \%)$ had blepharitis, $19(7.0 \%)$ had severe corneal exposure changes leading to infectious corneal ulcer and perforation in all of them. The pattern of microbial distribution in conjunctival discharge, exposure keratitis and corneal perforation are tabulated in Table 5. Pseudomonas aeruginosa was the most common microbe isolated in these cases suggesting hospital acquired infection.

\section{Discussion}

In this study, out of 272 samples collected from patients in ICUs, with the period of stay ranging from 1 to 13 days, $82.1 \%$ of the samples showed microbial growth with at least one microbe. $10.7 \%$ of the samples showed presence of more than one microbe in the culture. In the study by Saritas et al. [12], it was found that out of a total of 40 cultures from the conjunctiva, 17 (42.5\%) were positive for bacteria. In the study of Mela et al. [2], 54 (77\%) patients were colonized by at least one bacterial species other than normal flora within seven to 42 days.

The microbes isolated in our study were CONS, Staphylococcus aureus, Candida sp., Pseudomonas aeruginosa, Acinetobacter sp., diphtheroids and Enterococcus fecalis. Out of these, the most common isolated microbe was CONS (125, 55.5\% of isolates), among them majority of the 
samples were collected from medicine ICU. CONS was prevalent in sepsis/urosepsis and organophosphorus poisoning patients. Next to CONS, diphtheroids (33, 14.66\% of isolates) and Staphylococcus aureus (29, 10.6\% of isolates) were isolated from conjunctival swab cultures. In a study by Sahin et al. [13] similar findings like our study was observed. Apart from the normal conjunctival flora, Pseudomonas aeruginosa (5.33\%) was the most common isolated microbe which was prevalent largely in medicine ICU. Pseudomonas was also responsible for maximum no of (n $=8$ ) corneal perforation in our study population [14]. In the study by Chen et al. [1], three most common species were coagulase negative Staphylococcus sp., Staphylococcus aureus and Corynebacterium sp. which was similar to our study. In ICU patients, commensals such as coagulase negative Staphylococcus bacteria are potential pathogens and may exhibit virulence and cause sight threatening corneal affection when breached by dryness and exposure. In our study population exposure keratitis was the most common ocular finding and CONS was the most common isolated (n $=51$ ) organism in those patients.

The extensive use of antimicrobial agents in ICUs increases the risk of antimicrobial resistance. Of the CONS, $84 \%$ were penicillin resistant. Of these, $13.6 \%$ were resistant to both erythromycin and gentamicin, but were sensitive to tetracycline. Of these, $11.2 \%$ were resistant to erythromycin, gentamicin, cloxacillin and clindamycin. They were sensitive to tetracycline and vancomycin. In the study by Chalita et al. [15], the in vitro susceptibility of the most common ocular bacterial isolates to several antibiotics and changing trends in the antibiotic susceptibility in a 15 -year period was assessed. They found gentamycin, tobramycin, cephalosporine decreased in their in-vitro susceptibility testing to all pathogens.

A total of $81.8 \%$ of the diphtheroids and $100 \%$ of the Staphylococcus aureus were resistant to penicillin. Pseudomonas aeruginosa and Acinetobacter spp. did not show any antimicrobial resistance to the agents tested. One hundred percent of Enterococcus fecalis was resistant to both tetracycline and ampicillin. They were sensitive only to vancomycin.

Twenty-four (8.8\%) eyes had conjunctival discharge. The most common microbe isolated from these samples was Pseudomonas aeruginosa, followed by Staphylococcus aureus and Acinetobacter sp. In the study by Saritas et al. [12], the rate of mucopurulent or purulent secretion was
$36.25 \%$. Most of the specimens were positive for Staphylococcus epidermidis, Pseudomonas aeruginosa, Acinetobacter baumannii, Staphylococcus haemolyticus, Klebsiella, and Proteus mirabilis.

Nineteen (7.0\%) eyes had corneal perforation. The most common microbe isolated from these samples was Pseudomonas aeruginosa, followed by Acinetobacter sp. 220 $(80.9 \%)$ eyes showed exposure keratitis of varying degree. Microbes isolated from these samples were mostly commensals of the ocular surface. In the meta-analysis study by Rosenberg and Eisen [16], it was found that a total of $20 \%$ to $42 \%$ of patients in the ICU develop exposure keratopathy. In the study by Sivasankar et al. [10], exposure keratopathy was noted in $49(19.8 \%)$ of the 248 eyes in the study. In the study by Saritas et al. [12], keratitis was observed in $10 \%$ patients.

Saritas et al. [12] reported four patients with keratitis in their study in which two of them with both trachea and conjunctival cultures were positive for Pseudomonas aeruginosa and Acinetobacter baumannii. In the literature, Pseudomonas aeruginosa, Acinetobacter spp., Staphylococcus aureus, Staphylococcus epidermidis, Haemophilus influenzae, and Streptococcus species were shown to cause microbial keratitis $[2,5,17,18]$. Among these microorganisms, the most common one is Pseudomonas aeruginosa, which is highly virulent and causes a rapid onset devastating infection $[5,11,17,18]$. Pseudomonas aeruginosa was isolated in two cases and Acinetobacter calcoaceticus in one patient. In two of the subjects, the microorganisms were also isolated from the respiratory tract. In this study, while coagulase negative Staphylococcus sp., Staphylococcus aureus, and diphtheroids were isolated from patients with all primary disease, Pseudomonas aeruginosa and Acinetobacter sp. were mainly isolated in patients with either infectious causes or respiratory causes as primary illness. Presence of Enterococcus fecalis and Candida sp. were also mainly seen in patients with infectious causes, respiratory causes and patients with sepsis/urosepsis. Patients with organophosphorus poisoning, peritonitis and cardiac disease did not harbour Pseudomonas aeruginosa, Acinetobacter sp. or Enterococcus fecalis in their conjunctiva.

The strength of this study was the adequate power (large sample size) and the inclusion of patients of varying primary illnesses from medical and surgical ICUs. The shortcoming of the study was the absence of a control group 
(non-ICU such as ward patients) to know the pathogenicity and antibiotic sensitivity of the large numbers of commensals grown. Also, as it was a one-time study, follow-up of the culture positive patients after treatment with antibiotics, according to their sensitivity patterns, could not be evaluated.

Eye care in ICU patients cannot be overemphasized. In spite of prophylactic measures such as lubricant eye drops, antibiotic ointment and lid taping, sight threatening corneal infection can still occur. Knowing the common microbial colonization on the ocular surface in ICU patients and their sensitivity pattern will go a long way in taking appropriate prophylactic measures to contain infection.

Our study demonstrates that in ICU patients, the most common conjunctival flora is the normal flora such as coagulase negative Staphylococcus sp., diphtheroids and Staphylococcus aureus followed by pathogenic organisms such as Pseudomonas aeruginosa and Acinetobacter sp. More than $80 \%$ of the isolates were resistant to penicillin. Pseudomonas was the most common microbe isolated in cases with corneal perforation.

\section{Conflict of Interest}

No potential conflict of interest relevant to this article was reported.

\section{References}

1. Chen SY, Kuo MC, Wang PN, et al. Study of conjunctival flora in patients after peripheral blood stem cell transplantation and its correlation with tear secretion. Biomed $J$ 2012;35:493-9.

2. Mela EK, Drimtzias EG, Christofidou MK, et al. Ocular surface bacterial colonisation in sedated intensive care unit patients. Anaesth Intensive Care 2010;38:190-3.

3. Grixti A, Sadri M, Edgar J, Datta AV. Common ocular surface disorders in patients in intensive care units. Ocul Surf 2012;10:26-42.
4. Mercieca F, Suresh P, Morton A, Tullo A. Ocular surface disease in intensive care unit patients. Eye (Lond) 1999;13 (Pt 2):231-6.

5. Kirwan JF, Potamitis T, el-Kasaby H, et al. Microbial keratitis in intensive care. BMJ 1997;314:433-4.

6. Liesegang TJ. Physiologic changes of the cornea with contact lens wear. CLAO J 2002;28:12-27.

7. Hernandez EV, Mannis MJ. Superficial keratopathy in intensive care unit patients. Am J Ophthalmol 1997;124:212-6.

8. McHugh J, Alexander P, Kalhoro A, Ionides A. Screening for ocular surface disease in the intensive care unit. Eye (Lond) 2008;22:1465-8.

9. Imanaka H, Taenaka N, Nakamura J, et al. Ocular surface disorders in the critically ill. Anesth Analg 1997;85:343-6.

10. Sivasankar S, Jasper S, Simon S, et al. Eye care in ICU. Indian J Crit Care Med 2006;10:11-4.

11. Parkin B, Turner A, Moore E, Cook S. Bacterial keratitis in the critically ill. Br J Ophthalmol 1997;81:1060-3.

12. Saritas TB, Bozkurt B, Simsek B, et al. Ocular surface disorders in intensive care unit patients. ScientificWorldJournal 2013;2013:182038.

13. Sahin A, Yildirim N, Gultekin S, et al. Changes in the conjunctival bacterial flora of patients hospitalized in an intensive care unit. Arq Bras Oftalmol 2017;80:21-4.

14. Johnson JL, Sagraves SG, Feild CJ, et al. An unusual case of corneal perforation secondary to Pseudomonas keratitis complicating a patient's surgical/trauma intensive care unit stay. Am Surg 2000;66:972-4.

15. Chalita MR, Hofling-Lima AL, Paranhos A Jr, et al. Shifting trends in in vitro antibiotic susceptibilities for common ocular isolates during a period of 15 years. Am J Ophthalmol 2004;137:43-51.

16. Rosenberg JB, Eisen LA. Eye care in the intensive care unit: narrative review and meta-analysis. Crit Care Med 2008;36:3151-5.

17. Hilton E, Adams AA, Uliss A, et al. Nosocomial bacterial eye infections in intensive-care units. Lancet 1983;1:131820.

18. Ommeslag D, Colardyn F, De Laey JJ. Eye infections caused by respiratory pathogens in mechanically ventilated patients. Crit Care Med 1987;15:80-1. 